\title{
LFRic: meeting the challenges of scalability and performance portability in weather and climate models
}

Article

Accepted Version

Creative Commons: Attribution-Noncommercial-No Derivative Works 4.0

Adams, S. V., Ford, R. W., Hambley, M., Hobson, J.M., Kavčič, I., Maynard, C. M., Melvin, T., Müller, E. H., Porter, A. R., Rezny, M., Shipway, B. J. and Wong, W. (2019) LFRic: meeting the challenges of scalability and performance portability in weather and climate models. Journal of Parallel and Distributed Computing, 132. pp. 383-396. ISSN 0743-7315 doi: https://doi.org/10.1016/j.jpdc.2019.02.007 Available at https://centaur.reading.ac.uk/82138/

It is advisable to refer to the publisher's version if you intend to cite from the work. See Guidance on citing.

To link to this article DOI: http://dx.doi.org/10.1016/j.jpdc.2019.02.007

Publisher: Elsevier

All outputs in CentAUR are protected by Intellectual Property Rights law, including copyright law. Copyright and IPR is retained by the creators or other copyright holders. Terms and conditions for use of this material are defined in the End User Agreement. 


\section{CentAUR}

Central Archive at the University of Reading

Reading's research outputs online 


\title{
LFRic: Meeting the challenges of scalability and performance portability in Weather and Climate models
}

\author{
S. V. Adams ${ }^{\mathrm{a}}$, R. W. Ford ${ }^{\mathrm{b}}$, M. Hambley ${ }^{\mathrm{a}}$, J. M. Hobson ${ }^{\mathrm{a}}$, I. Kavčiča, \\ C. M. Maynard ${ }^{\mathrm{a}, \mathrm{c}}$, T. Melvin ${ }^{\mathrm{a}}$, E. H. Müller ${ }^{\mathrm{d}}$, S. Mullerworth ${ }^{\mathrm{a}}$, A. R. Porter ${ }^{\mathrm{b}}$, \\ M. Rezny ${ }^{\mathrm{e}}$ B. J. Shipway ${ }^{\mathrm{a}}$, R. Wong ${ }^{\mathrm{a}}$ \\ ${ }^{a}$ Met Office, UK \\ ${ }^{b}$ STFC Hartree Centre, Daresbury Laboratory, UK \\ ${ }^{c}$ Department of Computer Science, University of Reading, UK \\ ${ }^{d}$ Department of Mathematics, University of Bath, UK \\ ${ }^{e}$ Monash University, Melbourne, Australia
}

\begin{abstract}
This paper describes LFRic: the new weather and climate modelling system being developed by the UK Met Office to replace the existing Unified Model in preparation for exascale computing in the 2020s. LFRic uses the GungHo dynamical core and runs on a semi-structured cubed-sphere mesh. The design of the supporting infrastructure follows object-oriented principles to facilitate modularity and the use of external libraries where possible. In particular, a 'separation of concerns' between the science code and parallel code is imposed to promote performance portability. An application called PSyclone, developed at the STFC Hartree centre, can generate the parallel code enabling deployment of a single source science code onto different machine architectures. This paper provides an overview of the scientific requirement, the design of the software infrastructure, and examples of PSyclone usage. Preliminary performance results show strong scaling and an indication that hybrid MPI/OpenMP performs better than pure MPI.
\end{abstract}

Keywords: Separation of concerns; Domain specific language; Exascale; Numerical weather prediction

Email address: c.m.maynard@reading.ac.uk (C. M. Maynard) 


\section{Introduction}

The Met Office develops and maintains a large suite of numerical models that underpins its operational weather and climate research work. The Unified Model (UM) of the atmosphere is at the heart of this suite. The UM was developed in the late 1980s and introduced into operational use in 1990 [1]. Since its inception there has been a continuous drive to increase its complexity, accuracy and compute performance so as to deliver the Met Office's purpose which is to work at the forefront of weather and climate science for protection, prosperity and well-being.

In continuing to improve the Met Office's atmosphere model capability into the future, three challenges were anticipated.

Firstly, for many years much of the improvement in compute performance of the UM could be obtained by buying high performance computers with faster processors. However, clock speeds of new generations of standard CPUs are now often slower than the old. Scaling to more CPUs is limited by interprocessor communications, and the costs of powering machines with higher core counts is becoming unsustainable. All these factors challenge the ability to deliver higher resolution and more complex numerical models [2].

Secondly, a particular challenge for many atmosphere models, including the UM, relates to their use of the latitude-longitude (lat-lon) grid. The convergence of longitude lines at the poles causes numerical issues that make it hard to scale the model to higher core counts.

Thirdly, looking forward to the future, it is anticipated that HPC architectures will change radically, and that the diversity of architectures will increase. Even when porting from one CPU-based machine to another, there is a cost due to the need to re-tune the performance for the particular characteristics of the new machine. With the emergence of more diverse machines such as GPU-based machines, the cost will only get worse.

In collaboration with academic partners, the Met Office reviewed the options available to resolve these three issues [3]. The decision was to develop a new dynamical core called GungHo [4] written to run on an unstructured mesh that avoids the polar 
singularity problem. A new software infrastructure would be required as the UM infrastructure will not support an unstructured mesh. The architecture of the software infrastructure would impose a separation between scientific code and parallel systems code - code that supports parallelism on HPC machines - so as to reduce the cost and complexity of porting to new architectures. This paper describes the novel features of this new model infrastructure and experiences in developing scientific code within it, and provides some preliminary compute performance results.

\subsection{The LFRic Roadmap}

LFRic is the name given to the new atmospheric model and to the new software infrastructure which is being developed to host the GungHo dynamical core. LFRic is named after the pioneering weather forecaster Lewis Fry Richardson ( [5], [6]). The LFRic Roadmap comprises three phases: developing the first version of the software infrastructure to support the GungHo dynamical core; extension of the software infrastructure to support implementation of a full atmosphere model including physics codes; deployment of the model in operational trials in the lead up to its replacement of the Unified Model.

Currently LFRic is mid-way through Phase 2, which will end in 2020. The start of Phase 3 marks the point when the LFRic atmosphere model replaces the Unified model as the Met Office's main target for science and computational performance improvements. Phase 3 is expected to end with the deployment of the LFRic atmosphere model in the Met Office NWP Operational Suite towards the mid-2020s, following which the first climate configuration will be developed that uses LFRic.

At the core of the LFRic design, the software architecture of the natural science code imposes a separation of concerns between science code and code relating to parallelisation of the model. The architecture is called PSyKAl after the three layers it comprises: Parallel Systems or PSy layer code, Kernel code and Algorithm code. The architecture aims to separate scientific code in the algorithms and kernels from parallel code within the PSy layer. Metadata embedded in the scientific code is read by an application called PSyclone which automatically generates the PSy layer code. Initially, PSyclone has successfully converted serial code into OpenMP and MPI parallel code 
without changing any of the science code. The design is described in more detail in Section 3.

The PSy layer code generated by PSyclone includes calls to the LFRic software infrastructure. The LFRic infrastructure implements a data model that supports finite element, finite volume and finite difference model fields, domain decomposition of these fields on distributed memory platforms, and halo swaps of fields to support communication of information between distributed memory domains. This infrastructure is implemented in Fortran and uses Fortran 2003 constructs to impose the separation of concerns.

It is important to be clear that most of the focus of the project so far has been to support the science requirements of the GungHo dynamical core and atmosphere model, and to demonstrate the principle of the separation of concerns within the PSyKAl approach. Performance results provided in this paper are based on only parts of the scientific model (the dynamics and individual kernels) and are therefore preliminary. Currently development of the GungHo dynamics is still ongoing: important optimisations to its algorithmic performance such as provision of a multigrid preconditioner are not complete. Furthermore, while the LFRic infrastructure is capable of running physics codes copied from the UM, the codes are currently being pulled in as is, with no consideration of compute performance as yet.

Additionally, while targeting of future platforms is planned, even now the ability to test LFRic on some such machines is limited by lack of sufficient Fortran 2003 support by some compilers for the object-oriented features being exploited in the design of the LFRic infrastructure. While lack of compiler support has impacted the development and testing of LFRic, in the long run the $\mathrm{OO}$ design will better enable the infrastructure to develop and adapt without impacting scientific code.

The rest of the paper is organised as follows: The GungHo dynamical core and computational aspects are presented in Section 2. The software design for the separation of concerns and PSyKAl API are described in Section 3. The model infrastructure and use of libraries is discussed in Section 4. PSyclone, the code generator is presented in Section 5 and the package developed to apply linear solvers and preconditioners is presented in Section 6. Finally a scaling analysis is presented in Section 7 and conclu- 
sions drawn in Section 8.

\section{GungHo}

The dynamical core in an atmospheric model is responsible for simulating those fluid dynamical processes that are resolved by the underlying mesh. It is then coupled to a suite of subgrid physical parametrization schemes for processes that are not resolved (such as cloud microphysics and convection) and those that act as subgrid diabatic sources (such as longwave and shortwave radiative heating/cooling). For the purposes of this paper only the dry dynamical core without the subgrid processes is considered.

The GungHo dynamical core has been developed to replace the current ENDGame dynamical core in the UM. The GungHo model seeks to replicate the accuracy and stability of ENDGame whilst replacing the regular lat-lon grid with a quasi-uniform grid. This avoids the problems associated with the polar singularities in ENDGame resulting from the convergence of meridians at the poles. The mesh used to develop GungHo within LFRic is an equi-angular cubed-sphere, shown in Figure 1 [7]. This has the benefit of near uniform resolution across the globe and a maximum/minimum edge length of $\approx 1.3$ achieved by using only quadrilateral cells. However, this quasi-uniform mesh comes at the expense of losing orthogonality (the line between two neighbouring cells centres is not, in general, perpendicular to the edge shared between the two cells). Additionally, the two polar singularities of the lat-lon grid have been replaced by the eight corners of the cubed sphere, where only three cells meet at a vertex instead of the usual four. The lack of orthogonality and the presence of the corner singularities need to be taken into account when choosing an appropriate numerical method to avoid errors being dominated by the corners.

To generate the 3D mesh this $2 \mathrm{D}$ cubed-sphere mesh is extruded in the radial direction to form a spherical shell of cells. Where orography is present a terrain following radial coordinate is used such that every column contains the same number of cells. The horizontal mesh is treated as unstructured, such that it could easily be changed, for example, to an icosahedral mesh as considered in [8]. The vertical mesh is structured 


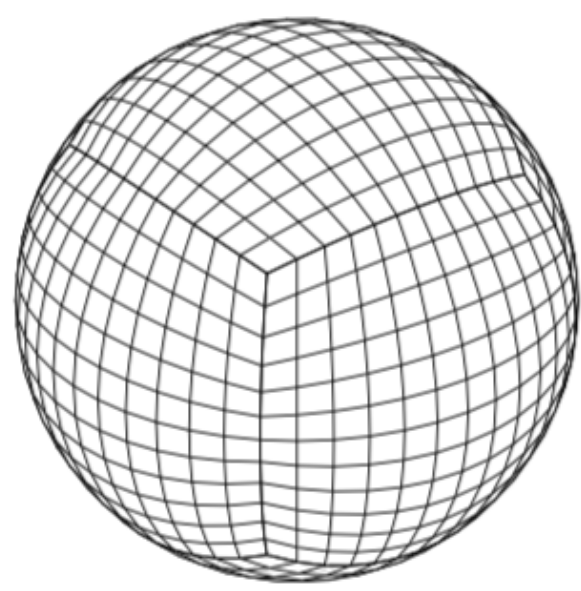

Figure 1: Equi-angular cubed-sphere mesh as used in GungHo with $12 x 12$ subdivisions per face, referred to as a $C 12$ mesh. This gives 864 columns of cells.

and directly addressed, so that a data point is addressed as $\operatorname{map}(d f, c o l)+k$, where $c o l$ is the horizontal index of the column and $k=0, \ldots$, nlayers- 1 is the index of the vertical layer; $d f$ is the index of a particular data point within the cell, allowing multiple data points within a single cell, and finally map is an array containing the address of data points at the bottom of the model column. Using an extruded mesh of this type with direct access and data contiguity in the vertical, the cost of the indirectly addressed horizontal index can be offset (see [9]) provided enough layers are used. Since the current UM uses $O(100)$ layers the cost of the indirection is minimal.

\subsection{Formulation}

The GungHo dynamical core solves the Euler equations for a perfect gas in a rotating frame

$$
\begin{aligned}
& \frac{\partial \mathbf{u}}{\partial t}=-(2 \boldsymbol{\Omega}+\nabla \times \mathbf{u}) \times \mathbf{u}-\nabla\left(\frac{1}{2} \mathbf{u} \cdot \mathbf{u}+\Phi\right)-c_{p} \theta \nabla \Pi, \\
& \frac{\partial \theta}{\partial t}=-\mathbf{u} \cdot \nabla \theta, \\
& \frac{\partial \rho}{\partial t}=-\nabla \cdot(\mathbf{u} \rho),
\end{aligned}
$$


the system is closed by the equation of state

$$
\Pi^{\frac{1-\kappa}{\kappa}}=\frac{R}{p_{0}} \rho \theta .
$$

These constitute a set of coupled non-linear Partial Differential Equations (PDEs) for the vector wind $\mathbf{u}$, the density $\rho$, potential temperature $\theta$ and Exner pressure $\Pi$. Additionally: $\boldsymbol{\Omega}$ is the rotation rate; $\Phi$ is the geopotential; $p_{0}$ is a reference pressure; $R$ is the gas constant; $c_{p}$ is the specific heat at constant pressure and $\kappa \equiv R / c_{p}$.

\subsection{Spatial Discretisation}

In order to maintain a similar accuracy to ENDGame on a quasi-uniform mesh a mixed Finite Element Method is used [10,11]. This gives the finite element equivalent of the C-grid-Charney-Phillips staggering $[12,13]$ used in ENDGame, but without relying on the orthogonality of the mesh for numerical consistency. The mixed FEM is very general in terms of the order of approximation and shape of the underlying mesh, allowing the method to be tailored to specific needs of the application. The mixed FEM involves defining a number of finite element spaces and differential mappings between them. The particular family of finite element spaces [14], for a given polynomial order $p$, used in GungHo are: $Q_{p+1}$ containing continuous point wise scalar quantities; $N_{p}$ containing circulation vectors that have continuous tangential components; $R T_{p}$ containing flux vectors that have continuous normal components; $Q_{p}^{D}$ containing discontinuous volume integrated scalars; along with a horizontally discontinuous vertically continuous space [11] for $\theta$ to mimic the Charney-Phillips grid staggering used in ENDGame. Here continuous means that neighbouring cells share degrees of freedom (hereafter dofs) located on the shared entities (e.g. faces, edges, vertices). In practice the lowest order spaces $p=0$ are used, the resulting location of the dofs for these spaces are shown in Figure 2.

The velocity field $\mathbf{u}$ is placed in the $R T_{0}$ space; the vorticity $\xi \equiv \nabla \times \mathbf{u}$ is placed in the

$N_{0}$ space; density, $\rho$, and Exner pressure, $\Pi$, are placed in the $Q_{0}^{D}$ space; as mentioned above the potential temperature, $\theta$, is placed in a scalar-space corresponding to the vertical components of $R T_{0}$ with dofs located in the centre of the top and bottom faces of a cell. See [4] for a full description of the discretisation used in the GungHo model. 


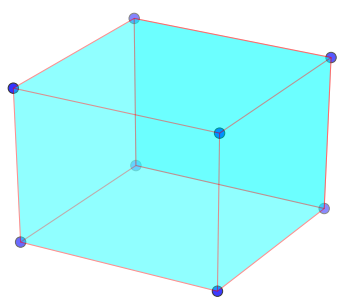

(a)

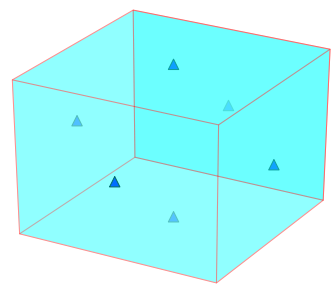

(c)

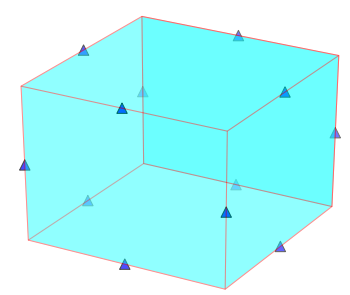

(b)

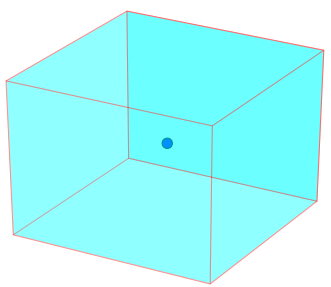

(d)

Figure 2: Location of degrees of freedom at $p=0$, (a) $Q_{1}$, (b) $N_{0}$, (c) $R T_{0}$ and (d) $Q_{0}^{D}$ finite element spaces. Circles correspond to scalar degrees of freedom and triangles to vector degrees of freedom.

\subsection{Advection}

In order to achieve accuracy similar to that of ENDGame it is important to have a high-order approximation to the scalar advective terms: $\mathbf{u} . \nabla \rho$ and $\mathbf{u} . \nabla \theta$. In ENDGame these are evaluated using a semi-Lagrangian formulation that involves computation of the trajectories that the fluid has taken over a timestep, followed by a high-order multi-dimensional interpolation of the scalar field to the origin of the trajectory. The non-local nature of the trajectory computation can lead to significant communication costs if the trajectory crosses over processor boundaries, which often happens near the poles of the lat-lon grid.

In GungHo this semi-Lagrangian scheme is replaced by an Eulerian finite-volume method of lines advection scheme that maintains inherent local conservation of mass. The method fits a high-order upwind polynomial over a number of neighbouring cells and evaluates this at a fixed point to compute the advective term. This only requires a local computation and the stencil is fixed, reducing the amount of computation needed. 
However, in order to obtain a stable scheme it is wrapped in a multi-stage evaluation, meaning that the advection update needs to be computed a number of times per iteration of the iterative-semi-implicit scheme (see Section 2.4); nominally 3 stages are used. Future work will evaluate the use of flux-form Semi-Lagrangian scheme (COSMIC) [15] to replace the current scheme. This carries many of the benefits of the semiLagrangian scheme used in ENDGame but without the communication costs (due to use of a quasi-uniform mesh) as well a simplification to the computation from using a dimensionally split formulation that only requires one-dimensional interpolation.

\subsection{Time-stepping}

As in ENDGame, to facilitate the use of long time-steps, a two time-level iterative semi-implicit time-stepping scheme is used. This requires, within each time-step, a non-linear Picard iteration to update the non-linear and advection terms and at each iteration a large sparse linear system is solved, which is formed by use of a quasiNewton method,

$$
\mathcal{L}\left(\mathbf{x}^{n}\right) \mathbf{x}^{\prime}=\mathcal{R}\left(\mathbf{x}^{(k)}\right),
$$

for the increment $\mathbf{x}^{\prime} \equiv \mathbf{x}^{(k+1)}-\mathbf{x}^{(k)}$ on the $k^{\text {th }}$ estimate from the Picard iteration of the prognostic variables $\mathbf{x} \equiv(\mathbf{u}, \theta, \rho, \Pi)$. The linear system $\mathcal{L}\left(\mathbf{x}^{n}\right)$ is chosen as to contain the terms necessary for stability of the fast acoustic and gravity waves as in [16] and is formed from a linearisation about the previous timestep fields $\mathbf{x}^{n}$. The method used to solve this linear system is detailed in Section 6. An overview of the time-step is given in Table 1.

\section{Separation of Concerns}

Science applications in general and weather and climate codes in particular are written in high-level languages such as Fortran or $\mathrm{C} / \mathrm{C}++$. Fortran is commonly employed for weather and climate codes as it is especially suited to numeric computation. Using such a high-level language, an algorithm is written to solve a mathematical problem without consideration for the processor architecture. The compiler generates machinespecific instructions and can, in principle, make optimisation choices to exploit the architecture of different processors. 


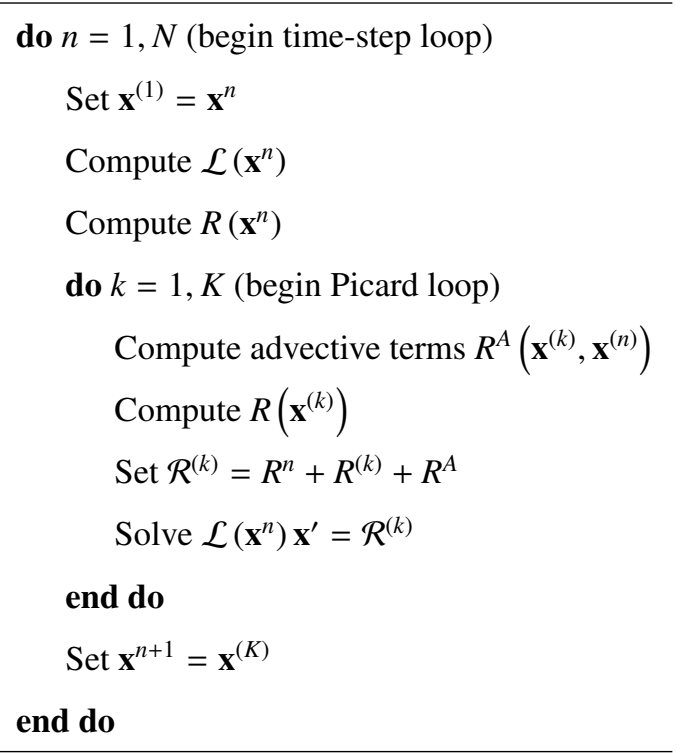

Table 1: Overview of a single timestep in the GungHo dynamical core, typically $K=4$.

This abstraction of a separation of concerns between mathematics code and machine code is powerful. It enables the portability of science code to different processor architectures, and allows the application to exploit a significant fraction of the peak performance of the processor. Whilst many science applications may contain code optimisations in performance-critical sections (for example, blocking or tiling loop nests to better utilise cache memory), in general these applications have relied on clock speed increases between processor generations to increase performance.

Increases in processor speed between generations ceased more than a decade ago due to the ending of Dennard scaling [17]. Instead, successive generations of processors have had increasing numbers of processor cores per socket. Science applications typically have already been adapted to run on multiple nodes to exploit supercomputers with the distributed memory and data parallelism typically expressed via MPI. Multicore nodes present additional opportunities and challenges to applications in terms of exploitation of shared address space within a node. Heterogeneous compute nodes such as CPU + GPU with distinct memory spaces require further additional programming models to enable proper exploitation of the available compute performance. 
A great number of programming models exist. For distributed memory there are, for instance, MPI and Partitioned Global Address Space (PGAS) languages, such as Co Array Fortran, Unified Parallel C, Chapel and GASPI. For threaded and sharedmemory parallelism there are directive-based solutions such as OpenMP and OpenACC as well as languages such as CUDA and OpenCL. However, the programming models lag behind in development of the rapidly evolving computer architectures. Particular models lack feature or processor coverage making the choice of programming model difficult. These issues are especially difficult for weather and climate applications with their long development cycles.

Many applications have adopted an MPI $+\mathrm{X}$ model, where $\mathrm{X}$ is one or more of the programming models mentioned in the previous paragraph. This is problematic for several reasons. The programming models are not only different, they are different types of model: languages, language extensions, libraries and directives, some of which are architecture specific. Worse, the interaction between them is outside the scope of any model and may, for example, depend on control by the batch scheduling system. The applications may require a different $\mathrm{X}$ for different architectures and even different data layouts and loop nest order. All these parallel and performance features break the data abstraction of the separation of concerns between the maths/science code and architecture-specific code.

The design for the LFRic model is based upon a computational science report from Phase 1 of the GungHo project [3]. By employing a layered software architecture, the complex parallel code can be kept separate from the science code. As outlined in Section 1, the software is separated into three layers. Algorithms are expressed as operations on global, data-parallel field objects, in the top algorithm layer. The middle PSy layer (Section 1) contains the looping over the horizontal field and is where the data parallelism is expressed. The bottom layer comprises the kernels which encode the operation invoked in the algorithm layer. Kernels are written to operate on a single vertical column of cells. Shown in Figure 3 is a schematic diagram of this layered software architecture. The red arrows indicate the control flow through the different layers.

The APIs between the layers are tightly controlled. The PSy layer can be called 


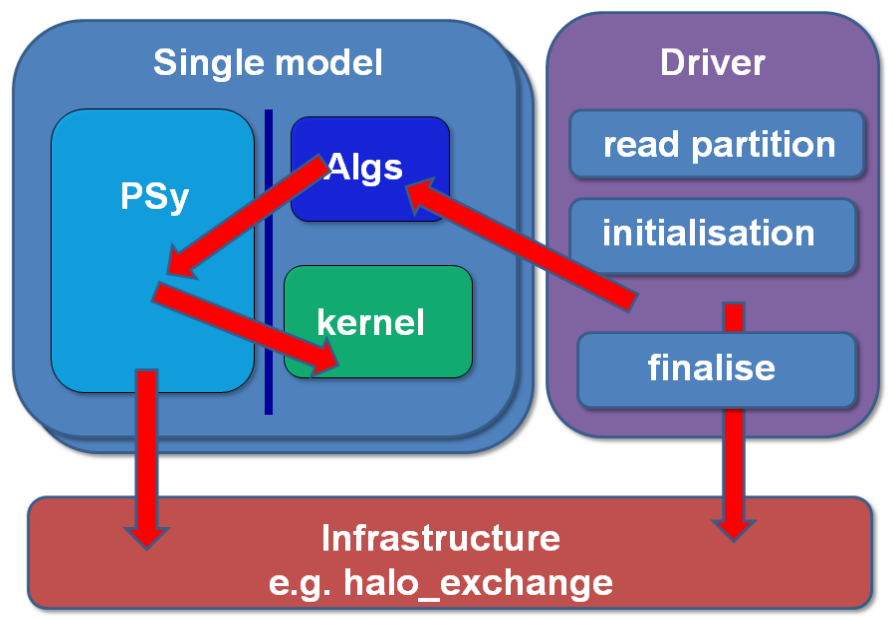

Figure 3: Schematic diagram of the PSyKAl layered architecture. A single model could be for example, the atmospheric model. The blue vertical line between the PSy layer and the Algorithm and Kernel layers represents a separation of the science and parallel code.

only from the algorithm layer by an invoke procedure call. The permitted arguments to these invoke procedures are a restricted set of LFRic objects, such as fields, that the kernels will operate on. The kernels can be called only from the PSy layer. The PSy layer code unpacks information from the LFRic objects including data arrays, scalars for loop bounds, and supporting arrays of both reals and integers for the Finite Element Method. The API is implemented using a Fortran 2003 object orientation coding style. By restricting the domain of the problem from all numerical mathematics to only that required (in the first instance) in the GungHo dynamical core it is possible to constrain the parallelism to the PSy layer.

Compilers in general have to be conservative about the assumptions they can make in order to guarantee correctness. However, a further advantage of the DSL approach is that developers can explicitly express the domain knowledge which is not possible in a standard, high level language. In the case of the LFRic model, data access descriptors are employed to say whether access to a field is read, write, readwrite or increment. The latter two discriminate between fields that do not share degrees of freedom between 
columns (e.g. density defined on $Q_{p}^{D}$ function space, see Section 2.2 for more details) and the ones that do (e.g. velocity defined on $R T_{0}$ space, Section 2.2$)$. This information is embedded in Fortran as part of the kernel. Other information encoded in this way is the loop iterator (for example, the choice to loop over horizontal cells), whether the kernel operates on a field which lives on a specific function space, whether any of the fields live on common function spaces and for FEM kernels whether the kernel uses any basis functions.

The restricted domain, strict control of the APIs between layers and, critically, the data access descriptors and other kernel metadata allow a set of rules to be derived to construct the PSy layer. The code itself can therefore be automatically generated, and a Python code parser, transformer and code generator called PSyclone has been written to generate it. More details of PSyclone can be found in Section 5. The generated PSy layer code is Fortran and contains calls to the LFRic infrastructure (see Section 4 for more details) to access functionality such as: MPI distributed memory parallelism, dereferencing the Fortran 2003 objects, looping over the horizontal mesh elements and calling the kernels themselves. PSyclone can also perform optional transformations to target other programming models such as OpenMP or OpenACC.

\subsection{Implementation}

The algorithm layer code is written in Fortran 2003. However, this code is not actually compiled; it is parsed and processed by PSyclone first. Most of the Fortran is left as written. The exception to this is the call to any invoke procedure. The invoke procedure does not exist as such in the source code. Instead, an invoke means execute these kernels looping over the chosen entities, hence instructing PSyclone on how to generate parallel code. PSyclone can parallelise this horizontal looping with MPI, OpenMP or both. Shown in the code fragment below is an invoke procedure call from the algorithm layer.

Listing 1: Code fragment showing an invoke procedure from the Algorithm layer

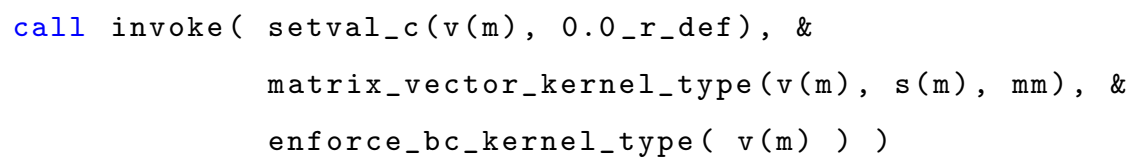


There are three kernels in this invoke. The first setval_c is an example of a pointwise kernel. The same operation, setting all the values of the field to the same scalar, is applied to each dof so no FEM structure is required. This is an example of a built-in operation generated in-place in the PSy layer by PSyclone. The second kernel is written as a call to the constructor of the kernel type, so that it is valid Fortran. The arguments to this call $\mathrm{v}$ and $\mathrm{s}$ are fields and $\mathrm{mm}$ is an operator, in this case a mass matrix.

PSyclone replaces the code for an invoke to the kernels with a call to a generated procedure in the PSy layer, with the fields and operators as arguments. Shown in Listing 2 is a code fragment from the generated PSy layer for the call to the matrixvector kernel in invoke shown in Listing 1.

Listing 2: Code fragment of the generated PSy layer with distributed memory support

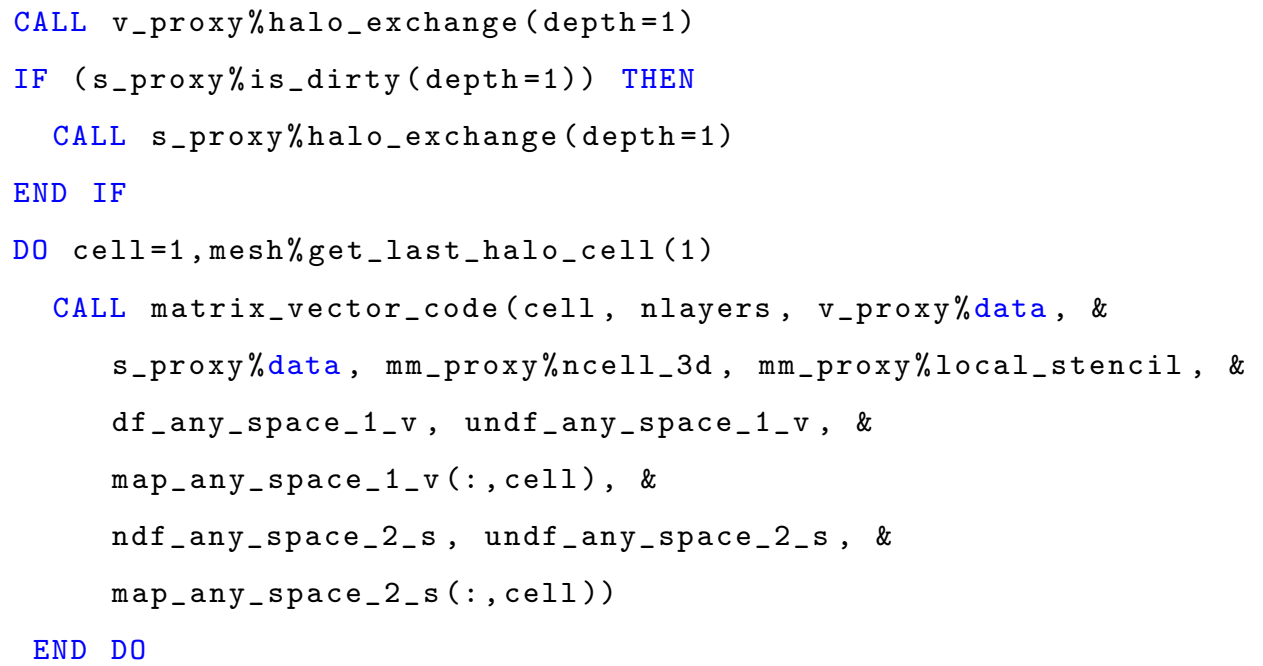

Lines 1-4 are the distributed memory calls to the infrastructure for a halo exchange and testing/setting flags to indicate whether a halo (and to what depth) has been updated. Lines 6-12 are the looping over the horizontal mesh and the procedure call to the kernel itself. The arguments are simple scalars for sizes and loop counters, the data arrays and (in this example) the indirection maps.

Shown in the code fragment below is the kernel metadata for the matrix-vector kernel invoked in the algorithm layer. 
Listing 3: Code fragment showing kernel metadata for the matrix-vector operator kernel

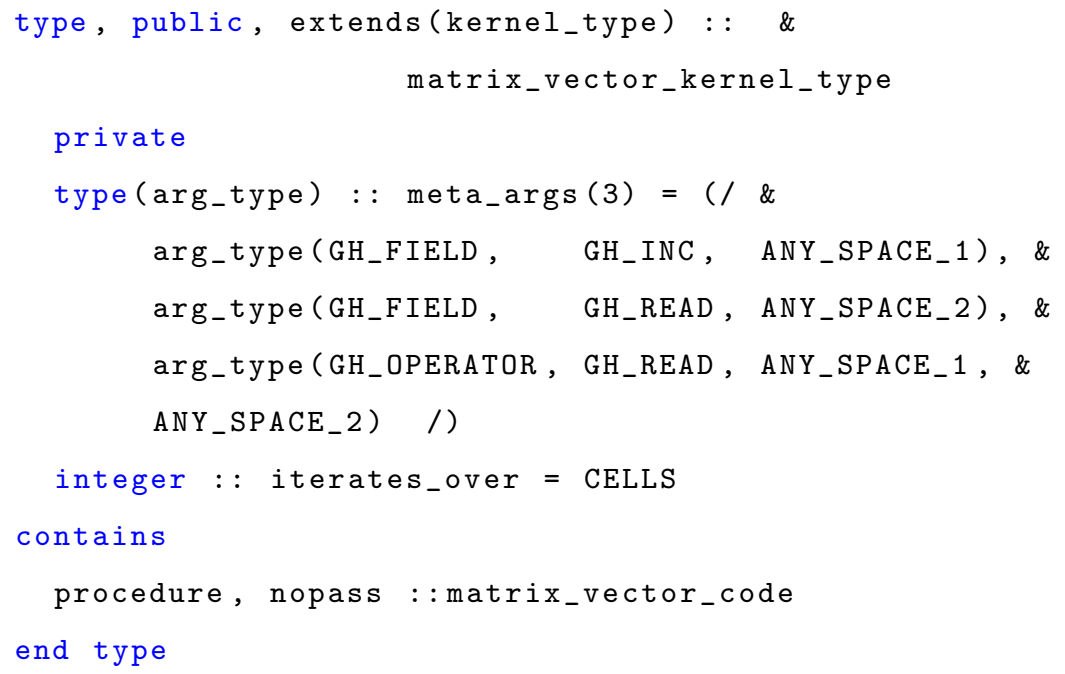

The metadata is embedded in Fortran so that no special comments or other mark-up is required. The access descriptors shown above are key to deriving the appropriate rules for generating the code in the PSy layer. The arg_type has three components. Firstly, the type of the data object, in this case fields and operators (an operator is a mapping between one function space and another). Secondly, the data access pattern for the data object. For example, GH_INC means the data is incremented. Thirdly, which function space the data lives on. In this case, the kernel is general and can operate on any function space, hence, the any_space_n. Whilst the $n$ can be any number, the function space of the first field must correspond to the first function space of the operator and the function space of second field must correspond to the second function space of the operator.

\section{Infrastructure}

As shown in Figure 3, the PSyKAl layered architecture is supported by the LFRic infrastructure. The LFRic infrastructure provides functionality such as distributed memory support (halo exchanges), colouring for OpenMP threading and the provision of loop bounds that the generated PSy layer will use. 


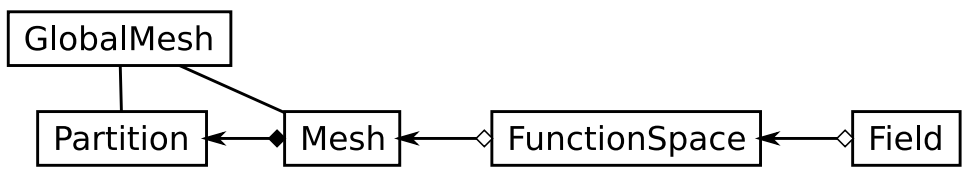

Figure 4: A Unified Modelling Language (UML) class diagram of the family of objects used in the LFRic infrastructure to support the field object.

Within the development of LFRic there has been a conscious effort to provide the infrastructure following an object-oriented approach. As such, much of the infrastructure is provided through a family of objects that ultimately support the field object that is used within the science code. The objects are shown in Figure 4. Two-dimensional topological and positional information about the global mesh is read into the global model object. This is passed to a partitioner to generate a partition object that describes what portion of the global mesh the local processing element will be working on. The global mesh and the partition objects are then combined to form a mesh object that extrudes the two-dimensional mesh information in the vertical for just the part of the mesh that will be used on the local processing element to form a local, threedimensional representation of the mesh. The way the data that is held within the field object relates to the mesh is described by the function space.

The LFRic infrastructure has been designed to be mesh-agnostic and thus most of the LFRic infrastructure supports a generic horizontally unstructured mesh. The partitioner is currently implemented to provide optimised partitioning specifically for cubed-sphere and planar meshes only. If a different mesh is required, the only infrastructure change required to support the new mesh is a relatively straightforward extension of the partitioner code.

Most of the infrastructure is accessed through the field object. So, for example if a halo exchange is required on a field, the halo exchange method on the field object is called. This infrastructure API is used by the PSy layer to access information and functionality. 


\subsection{Distributed Memory Optimisation}

Distributed memory parallelism is achieved by partitioning the full (global) domain into smaller sections (partitioned or local domains). Information is passed between the tasks through the use of halo regions. Data in these areas are provided from other partitions that own that section of the domain.

Performing halo exchanges is not unique to LFRic. Running large parallel weather and climate model codes has been done for many years, with halo exchanges being required to communicate updates between the partitions of data. However, the following aspects of the data model within the LFRic infrastructure make implementing distributed memory parallelism using halo exchanges particularly challenging:

1. LFRic is designed to support horizontally unstructured meshes, which means it has to support non-uniform shaped partitions with complex shaped halo regions;

2. Because the domain is horizontally unstructured, moving from one data point to a horizontally adjacent data point requires using a lookup table, which is slower than using direct addressing. In order to recover some code performance, fields are laid out in memory so that vertically adjacent data points are next to each other, and the looping structure within LFRic kernels is vertical looping innermost;

3. As noted in Section 2.2, the discretisation being used within LFRic leads to dof data being held on all the different entities within the mesh: cell volumes, faces, edges and vertices. A partitioning scheme that partitions the domain along cell boundaries leads to partitions that must share the dofs that are located on the boundaries of the domain. Having to assign ownership of these shared dofs to a particular partition adds to the complexity of setting up the halo exchanges.

The halo exchanges are achieved by passing information between processes using MPI. For performance and ease of use reasons, the MPI library is not called directly, instead a layer between the model and MPI is introduced. At initialisation time, the layer generates the communication routing tables required to perform a halo exchange. These tables are then reused every time a halo exchange is required. 
In order to generate the routing table, each rank needs to supply the following information to the library:

- A list of all the global dof ids of all the locally owned dofs;

- A list of all the global dof ids of all the dofs in the halos.

Initially, the LFRic project used the infrastructure library component of the Earth System Modelling Framework (ESMF) [18] to provide the layer between the model and MPI, however it was not particularly well-suited to the higher order finite-element fields used in LFRic. Although the routing tables it generated were optimal (the halo exchanges called during timestepping were very fast), only a small part of the ESMF framework was actually being used. This made it a rather heavyweight dependency. The design of the model framework to support the 'separation of concerns' led to all the halo exchange functionality being neatly encapsulated in the PSy layer. Therefore it was relatively easy to try a different library to provide the intermediate layer. A switch was made to use the YAXT (Yet Another eXchange Tool) [19] library. This provides halo exchanges during time stepping that are as fast as those provided by ESMF, via a lightweight library.

The API for supporting distributed memory optimisation is very simple. A halo exchange function is provided on every field and a flag is maintained by the infrastructure to track whether data held in the halo cells is up to date with the owner of that data ('clean' halos) or whether a parallel computation has updated the field on the owning partition and the local halos are out of date ('dirty' halos). When the PSy layer determines that a halo exchange may be required, it first checks the state of the halos and if (and only if) they are 'dirty' it calls the halo exchange functionality.

When the LFRic project was initiated, a survey of other models and systems found no other infrastructures or data models that would support the particular combination of higher order FEM methods and unstructured meshes. Since then, the ATLAS library [20] has emerged which in time may potentially be considered as an alternative data model capable of delivering LFRic requirements. 


\subsection{Shared Memory Optimisation}

As with all the parallelisation within LFRic, the shared memory parallelism is implemented by inserting directives in the PSy layer. The finite-element formulation makes use of data on mesh entities shared by more than one cell. Two different threads working on adjacent cells may try writing to a dof shared between the cells at the same time. Graph colouring is applied to the mesh so that no cells of any one colour share dofs. This means parallel threads can run over the whole set of cells within a particular colour and safely write dof values without contention. The information and functionality to support colouring within the shared memory parallelism is provided by the LFRic infrastructure.

When the mesh object is created, it is coloured by the infrastructure and information about the colouring is stored, so it can be used from within the PSy layer. The infrastructure provides the number of colours, the number of cells in each colour and which cells are present in each colour. Shared memory parallel directives are placed in the PSy layer and the looping can be made safe using the colouring information provided by the infrastructure.

\subsection{Parallel $\mathrm{I} / \mathrm{O}$}

A consequence of models running over many thousands of cores is a requirement for scalable parallel I/O. A computationally optimised model is of little practical operational use if $\mathrm{I} / \mathrm{O}$ then becomes a limiting factor. The LFRic project has decided to investigate options for $\mathrm{I} / \mathrm{O}$ alongside the computational and infrastructure development for three main reasons. Firstly, the requirement to be able to run science assessment jobs on large numbers of cores and obtain the output efficiently. Secondly, a key aim of LFRic is scalability and therefore it is helpful to be able to monitor the impact of $\mathrm{I} / \mathrm{O}$ on this as the infrastructure and science develops. Thirdly, LFRic is making some fundamental changes that impact the underlying mesh and this links to I/O in terms of input/output file formats. Having a concrete idea of what these file formats will be in the future enables LFRic to inform future users, internal and external to the Met Office.

Although developing a bespoke I/O system was a possibility, the decision was made early on to adopt an existing parallel I/O framework and leverage knowledge and ex- 
perience from the community; developing Earth System Models is becoming increasingly complex and challenging for any one organisation to develop and maintain all the required components [2]. As of early 2016, when the first scoping work for LFRic parallel I/O was being done, there were several existing parallel I/O frameworks and XIOS was selected as the prime candidate for evaluation [21]. A discussion of the other frameworks and why they were not considered can be found in [22].

XIOS was an obvious choice for LFRic as it was a mature framework, having had many years of development and was already in use in the weather and climate domain; for example, the Orchidee land surface model, the Dynamico dynamical core (part of the LMD-Z Atmosphere model) and the NEMO ocean model. NEMO is used in Met Office coupled models so there was some existing experience with XIOS. In terms of potential scalability, XIOS had also been proved to run successfully in models running on the order of 10,000 cores. Crucially for climate models, XIOS works with the OASIS coupling framework that is commonly used in coupled climate models including the UM.

The key features of XIOS can be summarised as follows:

1. Flexible definition of $\mathrm{I} / \mathrm{O}$ workflows via external XML files;

2. Client/Server architecture allowing asynchronous I/O servers on dedicated cores;

3. Sophisticated "in situ" post-processing via workflows defined in the XML file(s).

XIOS is written in $\mathrm{C}++$, but also provides a Fortran API to developers. It makes use of MPI-IO and the NetCDF4 and HDF5 libraries and handles unstructured and regular grids. XIOS is a client-server framework where $\mathrm{I} / \mathrm{O}$ servers are asynchronous processes buffering output requests from client processes. XIOS also has sophisticated post-processing functionality - e.g. for regridding, and computing time series and time averages. The output schedule and file format are defined by an XML file which hides a lot of complexity from the user.

Prior to 2016, XIOS only supported read and write of NetCDF file formats that follow the CF (Climate and Forecast) conventions [23]. As previously explained in Section 2, the LFRic implementation uses horizontally unstructured meshes and a FEM formulation, where variables are held on different elements of the mesh (vertices, edges 
or faces). Therefore, the $\mathrm{I} / \mathrm{O}$ system needs to handle data structured in this way. The UGRID-NetCDF format has been chosen as the main LFRic input mesh format and also output format for diagnostics and model dumps [24]. In the UGRID convention the topology of the underlying unstructured mesh is stored as well as data, and data can be defined on any of the mesh elements: vertices, edges, or faces. Working in collaboration with IPSL (Institut Pierre Simon LaPlace), the ability to write the UGRID format was added to XIOS in 2016 in order to support LFRic.

XIOS has been integrated into LFRic via a lightweight I/O interface so that (as far as possible) the underlying $\mathrm{I} / \mathrm{O}$ framework is hidden and could be replaced by an alternative. The I/O interface is designed to be compatible with the use of Fortran 2003 Object Orientation in LFRic. The field object (see Figure 4) contains read and write interface declarations that use Fortran procedure pointers to define I/O behaviours. When a field is created the behaviours are set by pointing to a specific reading or writing method. Writing a field just involves calling field\%write_field() - i.e., it is the responsibility of the field to write itself and how the writing is actually done is hidden.

More details of the experimental results of the LFRic-XIOS integration work can be found in [22] where preliminary results on I/O scalability (up to 13824 processors) and XIOS and Lustre performance tuning are presented. The conclusions of this work were that XIOS is an effective parallel $\mathrm{I} / \mathrm{O}$ framework that was technically straightforward to integrate into the existing LFRic infrastructure via the XIOS Fortran interface. Scaling experiments showed that XIOS performance was scalable with respect to increasing numbers of cores, even with minimal tuning.

\section{PSyclone}

As discussed earlier, PSyclone [25] is a domain-specific compiler which, given an algorithm and associated kernels, generates the code for the middle, PSy layer. Currently, each of these layers must be in Fortran and hence the compiler is embedded in Fortran. This makes the generated code easy to understand for domain scientists and means that existing debuggers and profilers can be used as if it were any other hand written Fortran code. The choice of Fortran is motivated in Section 3. However, 
there is nothing in the approach to prevent other languages being targeted in the future. PSyclone itself is written in Python.

PSyclone is heavily influenced by the OP2 system [26, 27]. However, PSyclone supports the specification of more than one kernel in a parallel region of code, compared with a limit of one for OP2, giving more scope for optimisation. Further, PSyclone takes responsibility for distributed memory parallelisation whereas it must be written manually by the application developer in OP2.

PSyclone is also designed to support a combination of kernels that are 1) written by the application developer and 2) provided by the system. It also allows optimisations to be applied by an HPC expert via a scripting interface, rather than necessarily automating the process. By way of contrast, in the related GridTools [28] and Firedrake $[29,30]$ approaches, the application developer specifies the mathematical operations (finite-difference stencils and finite element operations, respectively) in a high-level language and optimised code is generated automatically. Whilst this is a very powerful approach, it relies on the high-level language capturing all possible operations and for the system to produce highly optimised code automatically.

PSyclone, GridTools and Firedrake approaches are all based on the concept of (various flavours of) a Domain-Specific Language (DSL) for finite-difference and finiteelement applications. This is distinct from other, lower-level performance-portable abstractions, such as Kokkos [31] and OCCA [32] where the aim is to provide a language that permits an application developer to implement a kernel once and have it compile to performant single-node code on a range of multi- and many-core devices. These approaches compliment DSL's, in that DSL's could make use of them to generate single-node parallel code rather than using e.g. OpenMP or CUDA directly.

Lastly, CLAW [33] is a Fortran source-to-source translation tool designed to produce performance-portable Physics single-node code. As such it is complimentary to PSyclone and could be used to optimise PSyclone physics kernels that have been written by application developers.

Figure 5 shows the data flow within the PSyclone architecture. Starting from the left, the Algorithm is parsed (using fparser, a pure Python implementation of a Fortran parser) and any invoke procedure calls identified. The list of kernels (and their argu- 


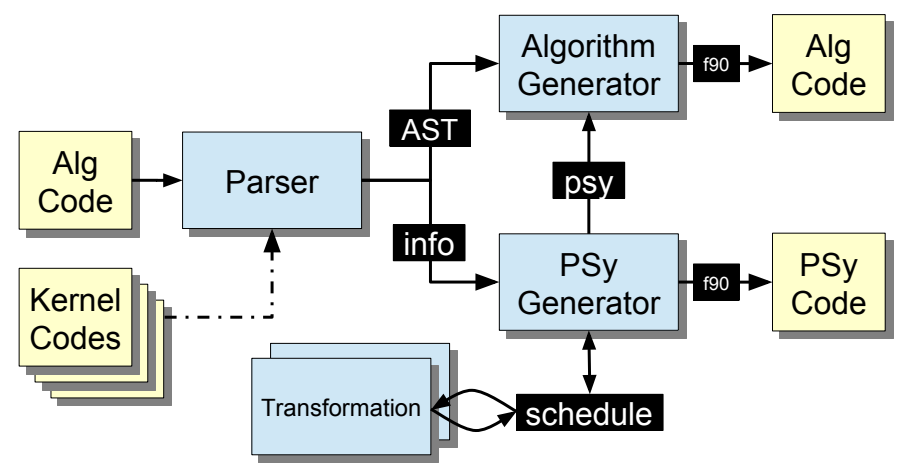

Figure 5: Data flow within the PSyclone architecture.

ments) associated with each of these calls is then stored. For each invoke, the Fortran modules containing the kernel code (as identified by the Fortran use statements) are parsed and the meta-data for each kernel extracted.

PSyclone must then perform two tasks. Firstly, generate an internal representation, known as a schedule, of a PSy layer routine for each invoke in the Algorithm. If distributed memory is specified then this schedule must include appropriate halo exchanges and global sums to ensure correct execution. Secondly, PSyclone must modify the supplied Algorithm code and replace each 'call invoke' with a call to the corresponding routine in the PSy layer. This latter task is achieved by modifying the fparser-produced Abstract Syntax Tree (AST) of the Algorithm and then re-generating Fortran from the new AST.

If no transformations are being applied (see below) to the PSy layer schedule(s) then all that remains is for the PSy generator to create Fortran code. The steps involved in generating a vanilla (un-optimised) PSy layer subroutine are as follows:

1. Generate the unique list of arguments required by all kernels in the invoke;

2. Generate Fortran which queries the LFRic infrastructure to get information on 
the sizes of the various iteration spaces (number of cells, vertical levels, dofs etc.);

3. Generate Fortran which queries the LFRic infrastructure to get the various lookup tables (e.g. for identifying the dofs belonging to a given cell);

4. Generate Fortran which dereferences the various field and operator objects to get the necessary data arrays;

5. For each kernel in the invoke

(a) Generate a loop over the correct iteration space (cells or dofs),

(b) Generate a call to the kernel subroutine with all necessary arguments.

Since the algorithm deals with global objects (fields, operators etc.) and a kernel deals with e.g. individual columns of cells (see e.g. Section 1), the PSy layer must also account for the fact that the global objects are in fact decomposed over MPI processes by the LFRic infrastructure. PSyclone must therefore ensure that global sums are placed appropriately and that the halos of any fields read by a given kernel are upto-date before that kernel is executed. Initially clean halos were implemented simply by generating code that, at run-time, marked any field written to by a kernel as having a dirty halo (using the LFRic infrastructure). Prior to each kernel call code was also inserted that, for each field with a halo access, performed a halo swap if the halo was dirty. Subsequently, dependence analysis has been added and now only those halo exchanges that are known to be required are inserted [25].

\subsection{Transformations}

The large variety in existing computer architectures, the number of different compilers and the fact that all of these are constantly evolving means that it is simply not possible to write or generate a single source code that will be (and continue to be) performance portable $[34,35]$. It is also very difficult to create a system that is automatically able to create performant code for such a range of conditions. PSyclone therefore seeks to be a tool for the HPC expert, enabling them to apply the optimisations that, thanks to their experience and knowledge, they know to be beneficial for a particular architecture and/or compiler. 
The application of optimisations is achieved by applying PSyclone-provided transformations to the schedule of an invoke. For instance, in order to parallelise a given schedule using OpenMP, PSyclone provides transformations to create a parallel region and introduce various forms of work-sharing construct. However, work-sharing of loops over cells for kernels which update quantities on horizontally continuous function spaces will result in race conditions where multiple threads attempt to write to the same dof on a shared mesh entity. It is therefore necessary to control the iteration space in order to prevent these race conditions and PSyclone provides a loop-colouring transformation for this purpose.

Although PSyclone transformations may be applied within a Python interactive session, they will typically be used during the compilation phase of a potentially large application. Therefore, PSyclone allows (Python) transformation scripts to be supplied on the command line. For example, to add OpenMP to the PSy layer, three transformations need to be applied. Firstly, the colouring transformation is applied to all loops in the schedule which contain fields on horizontally continuous spaces that are modified, Secondly, all loops, except loops over colours, have two OpenMP transformations applied. This script, shown in Listing 5 in the Appendix 8, has 17 lines of executable Python and is all that is required to apply OpenMP to the whole model. Note, PSyclone supports the application of transformations to any subset of a schedule and to any set of schedules so may be used for general whole-code optimisations and/or specific optimisations to particular code regions.

Applying the OpenMP transformations to the invoke call described in Section 3 results in the following generated PSy layer code shown in Listing 4.

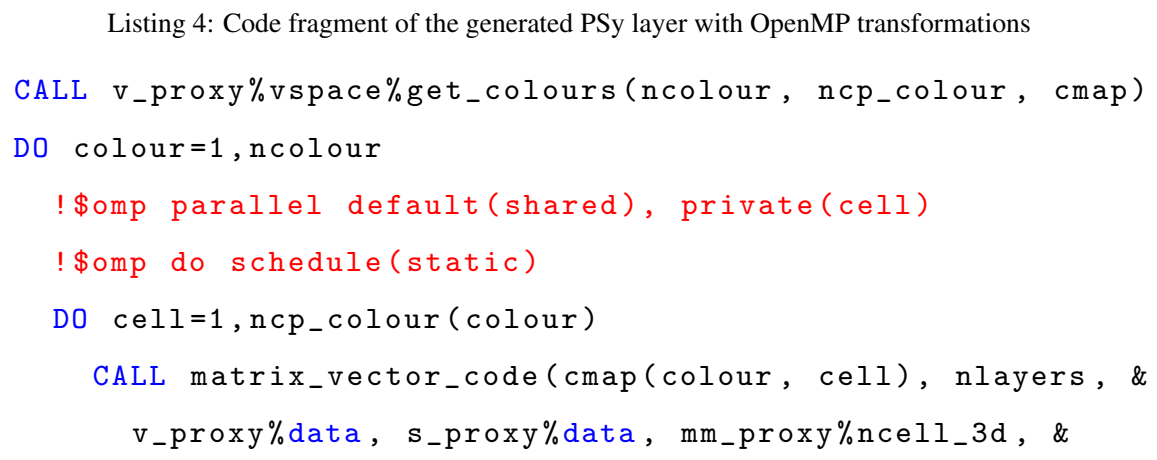


The transformation has resulted in a call to the LFRic infrastructure to obtain the colouring information, a loop over the number of colours, then the OpenMP workshare directives, which parallelise the loop over the cells and the colour.

\section{Linear solvers and preconditioners}

Iterative solvers for large sparse linear systems of equations are required in several places in the model. For example, since mass matrices in the finite element discretisation are not diagonal, they need to be inverted with a small number of iterations of a Krylov subspace method. More importantly, the semi-implicit time-stepping approach requires the solution of a very large sparse system for all prognostic unknowns in each step of the non-linear Picard iteration (Section 2.4). Since the system is ill-conditioned, it needs to be preconditioned efficiently. This is achieved by the (approximate) reduction to an elliptic system for the pressure, which itself is preconditioned with a tensorproduct multigrid algorithm [36] (see Section 6.2). To increase efficiency, the pressure preconditioner can be wrapped in its own iterative solver for the Helmholtz system. Note that in contrast to the approach already employed in the ENDGame model [16], an outer iteration over the full system is still required due to the non-diagonal nature of the finite element mass matrices. Altogether this results in a rather complex solver.

\subsection{Solver infrastructure}

To allow easy implementation of sophisticated nested iterative solvers and preconditioners, a dedicated abstraction was developed by using object-oriented features of 

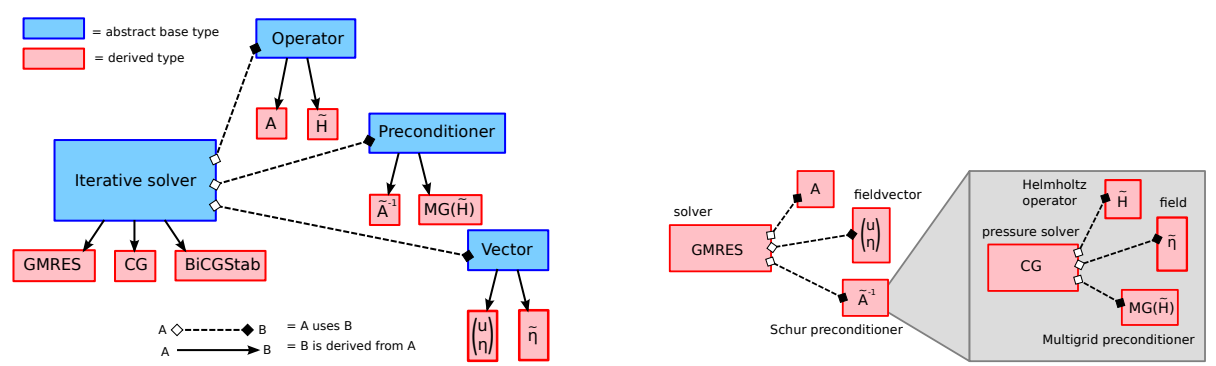

Figure 6: Derived class hierarchy for solvers and preconditioners in LFRic (left) and concrete implementation for the linear system in implicit time-stepping (right).

Fortran 2003. This approach follows similar design philosophies in widely used linear algebra libraries such as PETSc [37, 38] and DUNE-ISTL [39]. More specifically, the implementation in LFRic uses derived types which realise the following operations (see Fig. 6, left):

- Vector types which support common linear algebra operations such as AXPY $y \mapsto y+\alpha x$ and dot products $x, y \mapsto s=\langle x, y\rangle$. The most important vector-type is field_vector, which contains a collection of model fields;

- Linear operator types which implement the operation $x \mapsto y=A x$ for vectors $x$ and $y$;

- Preconditioners which approximately solve the system $P x=b$ for a given right hand side $b$ and some operator $P \approx A$;

- Iterative solvers which solve the system $A x=b$ with a Krylov-subspace method for a given right hand side $b$. Currently supported solvers include Conjugate Gradient, GMRES and BiCGStab.

Each of those types is derived from an abstract base type. The iterative solver types operate on generic vector types and are passed preconditioner and linear operator objects which adhere to the interface of their abstract base types. This implies that only one instance of a particular Krylov method has to be implemented in the code. Apart from avoiding code duplication, this increases reliability and maintainability, since only 
one instance of each solver has to be developed and tested. In addition, it allows easy "plug-and-play" to explore the space of all possible solver/preconditioner combinations to achieve optimal performance.

To solve a particular problem, the user has to develop bespoke derived types for the corresponding linear operator and preconditioners. Note that the apply() methods of those derived types contain invoke calls to kernels, which guarantees optimal performance of the entire model. For example, to construct a solver for the implicit linear system which is inverted in every semi-implicit time-step, the user implements the following objects (see Fig. 6, right):

- A linear operator type which applies the full linear system to all components of a field_vector;

- A preconditioner type which reduces the full linear system to the (approximate) Schur-complement in pressure space by lumping the velocity mass matrix; this preconditioner then calls the solver for the pressure (Helmholtz) system;

- A linear operator type which applies the Helmholtz operator for the pressure system;

- A preconditioner type which approximately inverts the Helmholtz operator (see Section 6.2).

Once implemented, those linear operators/preconditioners need to be passed to suitable existing linear solvers.

In addition to this more traditional approximate Schur-complement approach for solving the full linear system, the development of solvers based on a hybridisation approach is currently being explored [40, 41]. Since an exact Schur-complement can be formed in this case, hybridisation avoids the expensive iteration over the full linear system, and potentially leads to significant improvements in performance.

\subsection{Preconditioner}

To precondition the strongly anisotropic Helmholtz operator for the pressure system, the tensor-product multigrid approach in [36] is being developed for use in LFRic. 
This is a more advanced solver than the current tridiagonal vertical-only preconditioner in the ENDGame model. The multigrid algorithm currently being developed in LFRic has been tested extensively for representative elliptic equations in atmospheric modelling in $[42,43]$, including a mixed-finite element discretisation of a linear gravity wave system in [44]. The key idea to address the strong vertical anisotropy due to the high-aspect ratio of the domain is to combine vertical-only smoothing (line relaxation) with a horizontal multigrid hierarchy. To allow the easy construction of the verticalonly operators in the Schur-complement from the finite element discretisation of the full equations, a suitable operator algebra was developed in [44] and tested in the Firedrake library. For horizontally discontinuous function spaces (such as the pressure space, $Q_{0}^{D}$, and the vertical-only components of the $R T_{0}$ velocity space, see Section 2.2 for more details), operators can be partially assembled into a matrix type which stores all couplings within one vertical column. Matrices of this type can be multiplied, added and, most importantly, inverted in the tridiagonal solvers which realises the vertical line relaxation. This allows the high-level construction of the building blocks required for the Helmholtz solver and preconditioner. The same data structures were implemented as derived Fortran 2003 types in the LFRic code and form the building blocks of the tensor-product multigrid preconditioner for the elliptic Helmholtz system.

Compared to simpler preconditioners, which do not combine vertical line relaxation with a horizontal multigrid hierarchy, the tensor-product multigrid approach typically reduces the solution time of the pressure system by a factor of at least two [42, 44]. However, since the Helmholtz system contains a zero-order term, only a small number ( $\approx 3-4$ ) of multigrid levels is required (independent of the grid resolution). This greatly increases scalability since it avoids global couplings which arise on the coarsest level.

\section{Scaling}

A key goal of LFRic and GungHo is scalability to meet run-time requirements. The use of an unstructured mesh from GungHo and the solver construction described in Section 6 should enable the LFRic model to scale to a very large degree of parallelism. However, compiling some of the Fortran 2003 object-oriented constructs necessary to 


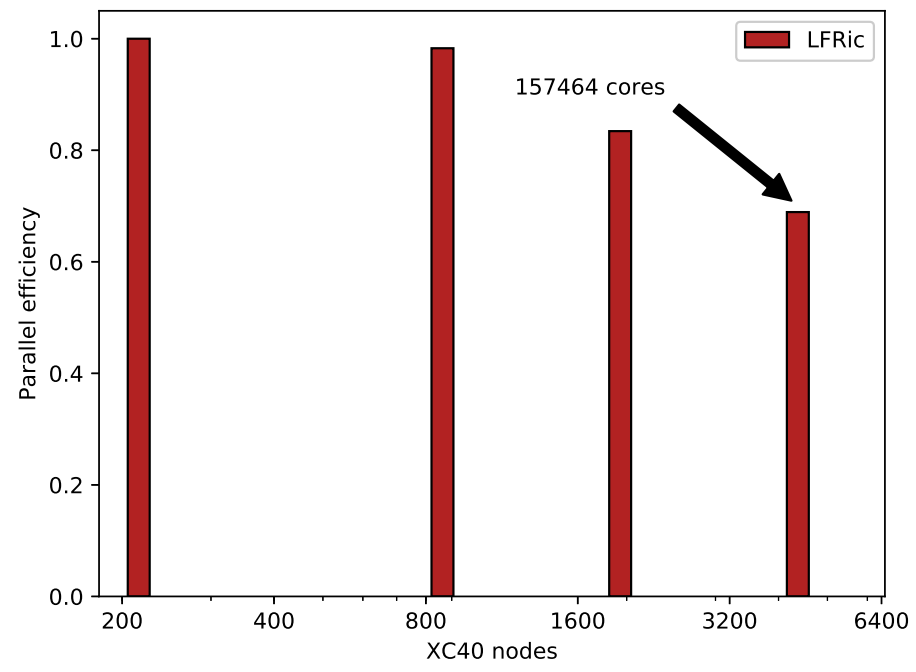

Figure 7: Strong scaling of the parallel efficiency of the LFRic time-steps compared to 216 nodes. N.B. The $\mathrm{x}$-axis shows a logarithmic scale

build such a solver infrastructure is a challenging task for most compilers which has delayed implementing such a solver. This remains a work in progress.

The February 2018 release of LFRic contains a simpler solver. This employs a Krylov iterative GCR algorithm on the mixed system of velocity, potential temperature, density and Exner pressure. This is preconditioned by an approximate Schurcomplement for the Exner pressure which uses lumped mass matrices. The pressure (Helmholtz) system is solved with BiCGstab and preconditioned by a vertical only tridiagonal solver. Both mixed and Helmholtz solvers use a relative tolerance of $10^{-6}$.

Shown in Figure 7 is the parallel efficiency of the LFRic time-step for a very high resolution model run. The mesh is a $C 1944$ cubed-sphere with 30 levels, where each panel has $1944 \times 1944$ cells. This roughly corresponds to a $5 \mathrm{~km}$ global resolution. The science configuration is the baroclinic wave test [45] with a 75 second time-step. The code was compiled to production level (-03) with the Intel 17 compiler. The model was run in hybrid mode with both MPI and OpenMP on the Met Office Cray XC40. Each node comprises of dual socket Intel Xeon (Broadwell) 18-core processors. The 
affinity was set to be three MPI ranks per socket with six OpenMP threads per MPI rank.

The model shows good strong scaling out to a very large number of nodes, 4374 , which is 157366 cores. Scaling starts to drop off at this scale to just below $70 \%$ compared to 216 nodes. Here, the local volume has been reduced to $12 \times 12$ with only 30 levels that is only 4320 dofs per unit of parallelism for the pressure space. The ratio of communication to computation cost will become worse if the problem is scaled further. The number of iterations for the solver is relatively large and with multiple solves per time-step and five global sums per iteration of the BiCGStab algorithm, the cost of the global sums becomes prohibitive.

At this stage it would be difficult to make a meaninful comparison to the performance of the current UM. The physical parameterisation schemes are, as yet, mostly absent from LFRic. Moreover, the UM has been highly optimised for the CPU architecture. The unstructured mesh will give LFRic the algorithmic scaling advantage but the absolute performance, i.e. wall-clock time, will still be inferior. However, the new solver implementation will enable better scaling by reducing the number of Krylov solver iterations required by using a much more efficient preconditioner. This will also improve the absolute performance, i.e. reduce run-time. Until the solver is closer to being algorithmically optimal it does not make sense to apply further computational optimisations.

Shown in Figure 8 is the effect of using OpenMP on scaling for 50 time-steps of the baroclinic wave problem, in this case with the May 2018 release of LFRic. The code was again compiled with the Intel 17 compiler. The model employs redundant computation into the halos. Each processor computes the contribution to dofs that reside on a shared mesh entity (such as a face) which belong to a cell adjacent to a halo cell from both the "owned" cell and the halo cell. Thus avoiding communication that would otherwise be required to correctly calculate the contribution of neighbour cells to that dof. Comparing MPI only to the hybrid mode, each processor in the former regime has more work to do in the case of redundant computation from the scaling of volume to surface area. The mixed-mode has fewer cells to redundantly compute than MPI only. Choosing redundant computation as a communication reduction algorithm 


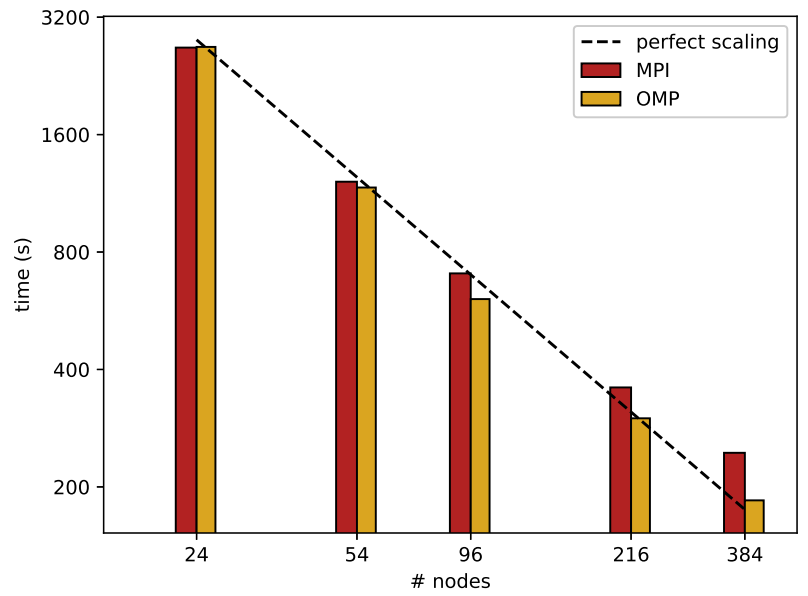

Figure 8: a) Log-log plot of strong scaling of 50 time-steps of the baroclinic wave problem on a cubed sphere mesh, C576, with 30 levels from the May 2018 release. The red bars, labelled MPI, show MPI only, i.e. 36 MPI ranks per node. The yellow bars, labelled OMP, show mixed-mode, with 6 MPI ranks per node and 6 OpenMP threads per MPI rank. The dashed line shows perfect scaling and is drawn to guide the eye.

favours threading; hence OpenMP is faster and scales better.

\section{Discussion and Conclusion}

This paper describes the novel features of the design of the new LFRic model and infrastructure, and the GungHo dynamical core within it. The challenges of GungHo's higher order FEM approach on a horizontally unstructured mesh essentially resulted in a requirement to develop a new infrastructure from scratch. This requirement provided an opportunity to develop a Domain Specific Language by separating the concerns of the science code from the parallel code. A domain specific compiler called PSyclone has been developed to automatically generate the OpenMP and MPI parallel code required to deploy the application on an HPC machine.

DSLs also feature heavily in other work seeking to make weather and climate models exascale ready. In contrast to this work, they are only part of the solution. For example, MeteoSwiss have ported the COSMO model to run on GPUs, by rewriting 
the dynamical core code using a DSL (STELLA). Other parts of the code (e.g. physics) have been instrumented with compiler directives [46]. CSCS (the Swiss National Supercomputing Centre) have made significant progress in porting the ICON dynamical core to run on GPUs by instrumentation with OpenACC directives and several physics schemes have been ported using a combination of OpenACC and the CLAW source to source translation tool. To further this effort, the ENIAC (ENable Icon on a heterogeneous ArChitecture) project seeks to make the ICON model exascale ready using a similar strategy of tools (the GridTools DSL and the CLAW source to source translator) and OpenACC directives [47]. The E3SM (Energy Exascale Earth System Model) project (a collaboration between 7 National US laboratories) [48] has ported HOMME (the dynamics/transport component of the Community Earth System Model) to a single source version (HOMMEXX) able to run on MIC, CPU and GPU architectures using Kokkos [31].

In summary, the design of LFRic has so far proven to be successful. The introduction of PSyclone has enabled seamless switching from serial to parallel running. Having initially being run in serial for many months while the first version of PSyclone was developed, it was possible to run in parallel (both MPI and OpenMP) on 220,000 cores of the Met Office Cray XC40 within two weeks of the full PSyclone functionality becoming available.

The GungHo dynamical core is routinely run in a range of science configurations and several scientists are currently developing code within the system. For the most part, scientists develop algorithms and kernels within the existing infrastructure and with auto-generated PSy layer code. For some newer developments the PSy layer code is hand-written while new features are added to PSyclone. A clean separation between the science code and the PSy layer code has been maintained throughout.

The need for PSyclone and the LFRic infrastructure to continuously evolve in the light of new and changing science requirements underlines an important point: the 'separation of concerns' that has enabled the science code to be kept isolated from the parallel systems code does not mean that scientists and engineers can work in isolation. In fact, the close ties between the scientists and software engineers has been a key part of the success of the approach. 
The scaling performance of the GungHo dynamical core is promising. While significant improvements in the algorithmic design are required to deliver better throughput of the model, the application (with I/O capability switched off) scales well, with scaling tailing off at around 160,000 cores due to the local volume of current configurations becoming very small. One of the first improvements to the algorithmic performance is anticipated to come from application of a geometric multigrid solver within the new solver infrastructure discussed in the paper.

Currently, the generation of MPI and OpenMP code is supported by PSyclone. The approach of computing results into the halo redundantly so as to reduce interprocessor communications helps the performance of deployments with more OpenMP threads per MPI rank (for a given total core count) because the larger domain of the highthread runs means that the relative size of the halos, and therefore the relative amount of redundant computation, is smaller. Moreover, PSyclone has been developed with the ability to add transformations that increase the amount of redundant computation so as to reduce the number of halo swaps, to re-order kernels and to loop-fuse kernels to provide a performance benefit. Work is also underway to support OpenACC transformations which can then be applied without having to re-write the science code.

The overall design has sought to carefully modularise functionality. A benefit of this is to enable trialling of different externally sourced libraries. An example of this was when the ESMF library was replaced by the YAXT library in a short period of time.

Development of the core LFRic infrastructure and PSyclone is still in its early days. LFRic is currently written to support any order of finite element method on a range of different horizontally unstructured meshes. Should the GungHo science converge on a particular mesh or set of meshes, and on a particular FEM order it will likely be possible to refactor the underlying LFRic data model to support more efficient access to the data.

As well as continuing the technical capabilities of LFRic, currently, sub-grid physics processes are being added to the GungHo dynamical core implementation of LFRic with a view to steadily building up to a fully-specified NWP model around 2020. Assuming the scientific and computational performance results are acceptable it will 
likely go head-to-head with the Met Office UM for 3-4 years while it is further enhanced and optimised, eventually replacing the Unified Model atmosphere at the core of the Met Office operations.

\section{Acknowledgements}

The original designs for the computational infrastructure and the GungHo formulation arose from the GungHo project. The contribution of GungHo Consortium members as well as UM Partnership collaborators and GHASP team members is gratefully acknowledged.

\section{Appendix}

Listing 5: Python script for applying transformations to a PSyclone schedule.

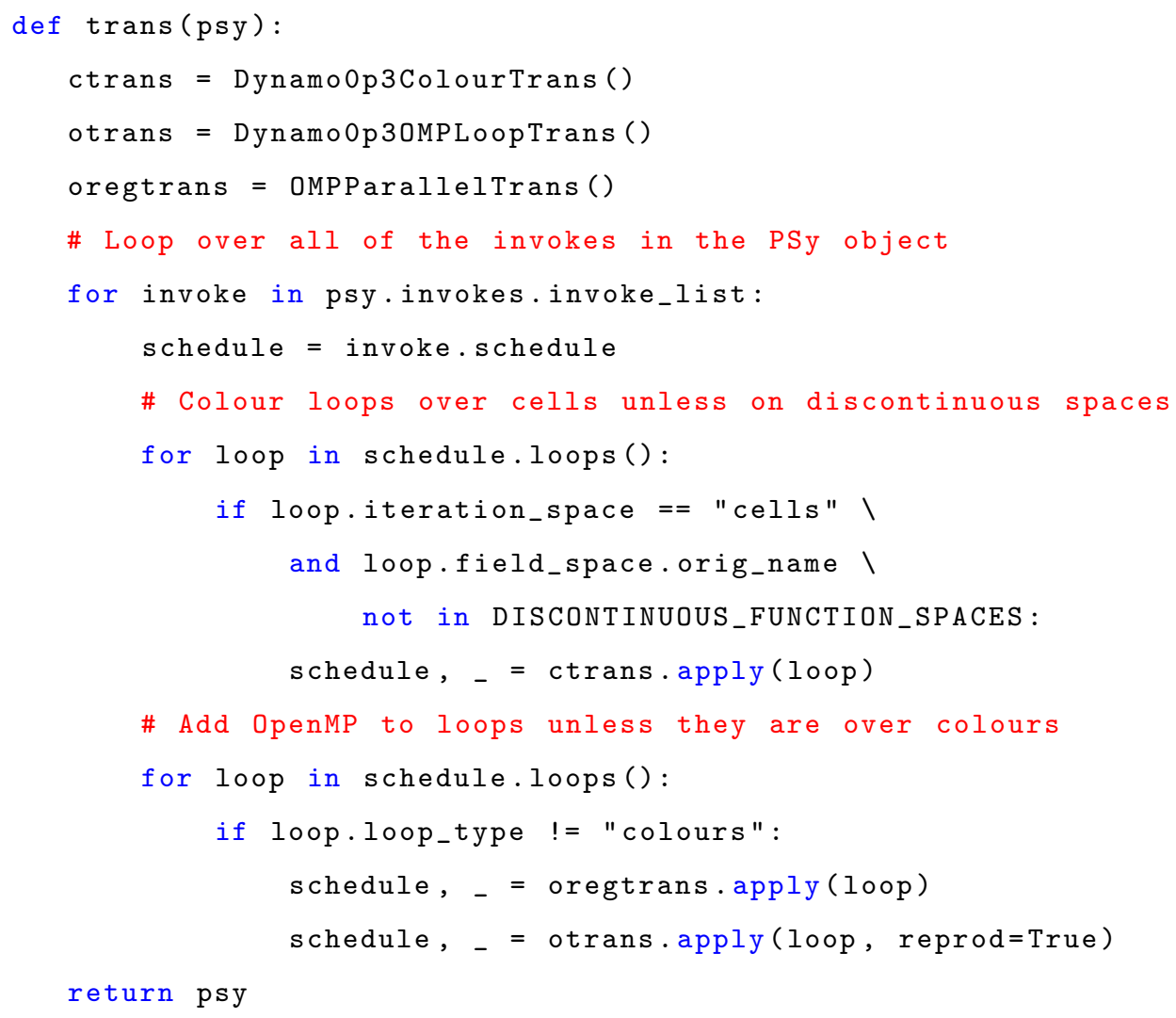




\section{References}

[1] D. Walters, I. Boutle, M. Brooks, T. Melvin, R. Stratton, S. Vosper, H. Wells, K. Williams, N. Wood, T. Allen, A. Bushell, D. Copsey, P. Earnshaw, J. Edwards, M. Gross, S. Hardiman, C. Harris, J. Heming, N. Klingaman, R. Levine, J. Manners, G. Martin, S. Milton, M. Mittermaier, C. Morcrette, T. Riddick, M. Roberts, C. Sanchez, P. Selwood, A. Stirling, C. Smith, D. Suri, W. Tennant, P. L. Vidale, J. Wilkinson, M. Willett, S. Woolnough, P. Xavier, The Met Office Unified Model Global Atmosphere 6.0/6.1 and JULES Global Land 6.0/6.1 configurations, Geoscientific Model Development 10 (4) (2017) 1487-1520. doi:10.5194/gmd-101487-2017.

URL https : //www.geosci-model-dev.net/10/1487/2017/

[2] B. N. Lawrence, M. Rezny, R. Budich, P. Bauer, J. Behrens, M. Carter, W. Deconinck, R. Ford, C. Maynard, S. Mullerworth, C. Osuna, A. Porter, K. Serradell, S. Valcke, N. Wedi, S. Wilson, Crossing the Chasm: How to develop weather and climate models for next generation computers?, Geosci. Model Dev. 11 (2018) 1799-1821. doi:10.5194/gmd-11-1799-2018.

URL https : //www.geosci-model-dev.net/11/1799/2018/

[3] R. Ford, M. Glover, D. Ham, C. Maynard, S. Pickles, G. Riley, GungHo Phase 1: Computational science recommendations, Technical report, Met Office (2013).

URL https://www.metoffice.gov.uk/binaries/content/assets/ mohippo/pdf/8/o/frtr587tagged.pdf

[4] T. Melvin, T. Benacchio, B. Shipway, N. Wood, J. Thuburn, C. Cotter, A mixed finite-element, semi-implicit finite-volume discretisation for atmospheric dynamics: Cartesian geometry, Q. J. Roy. Meteorol. Soc.In preparation.

[5] P. Lynch, The Emergence of Numerical Weather Prediction: Richardson's Dream, Cambridge University Press, 2006.

[6] P. Lynch, Richardson's fantastic forecast factory, european meteorological society website, https://www.emetsoc.org/resources/rff/, accessed 12 October 2018. 
[7] R. D. Nair, S. J. Thomas, R. D. Loft, A discontinuous Galerkin transport scheme on the cubed sphere, Monthly Weather Review 133 (4) (2005) 814-828. doi:10.1175/MWR2890.1.

URL https : //doi.org/10.1175/MWR2890.1

[8] A. Staniforth, J. Thuburn, Horizontal grids for global weather prediction and climate models: A review, Q. J. Roy. Meteorol. Soc. 138 (2012) 1-26. doi:10.1002/qj.958.

[9] A. E. MacDonald, J. Middlecoff, T. Henderson, J.-L. Lee, A general method for modeling on irregular grids, The International Journal of High Performance Computing Applications 25 (4) (2011) 392-403. doi:10.1177/1094342010385019.

URL https : //doi.org/10.1177/1094342010385019

[10] C. J. Cotter, J. Shipton, Mixed finite elements for numerical weather prediction, J. Comput. Phys. 231 (2012) 7076-7091.

[11] A. Natale, J. Shipton, C. J. Cotter, Compatible finite element spaces for geophysical fluid dynamics, Dynam. Stat. Climate Sys. 1 (1). doi:10.1093/climsys/dzw005.

[12] J. G. Charney, N. Phillips, Numerical integration of the quasi-geostrophic equations for barotropic and simple baroclinic flows, J. Meteor. 10 (2) (1953) 71-99.

[13] A. Arakawa, V. R. Lamb, Computational design of the basic dynamical processes of the UCLA general circulation model, in: J. Chang (Ed.), General Circulation Models of the Atmosphere, Vol. 17 of Methods in Computational Physics: Advances in Research and Applications, Elsevier, 1977, pp. 173-265. doi:https://doi.org/10.1016/B978-0-12-460817-7.50009-4.

URL http://www.sciencedirect.com/science/article/pii/ B9780124608177500094

[14] D. Boffi, F. Brezzi, M. Fortin, Mixed Finite Element Methods and Applications, Vol. 44, Springer, 2013. 
[15] B. P. Leonard, A. P. Lock, M. K. MacVean, Conservative explicit unrestricted-time-step multidimensional constancy-preserving advection schemes, Mon. Weather Rev. 124 (11) (1996) 2588-2606. doi:10.1175/15200493(1996)124;2588:CEUTSM $\_2.0 . C O ; 2$.

[16] N. Wood, A. Staniforth, A. White, T. Allen, M. Diamantakis, M. Gross, T. Melvin, C. Smith, S. Vosper, M. Zerroukat, J. Thuburn, An inherently massconserving semi-implicit semi-Lagrangian discretization of the deep-atmosphere global non-hydrostatic equations, Q. J. Roy. Meteorol. Soc. 140 (682) (2014) 1505-1520. doi:10.1002/qj.2235.

URL http://dx.doi.org/10.1002/qj.2235

[17] R. H. Dennard, F. H. Gaensslen, V. L. Rideout, E. Bassous, A. R. LeBlanc, Design of ion-implanted MOSFET's with very small physical dimensions, IEEE J. SolidState Circuits 9 (5) (1974) 256-268. doi:10.1109/JSSC.1974.1050511.

[18] ESMF project website, http://www.earthsystemmodeling.org, accessed 23 July 2018.

[19] YAXT project website, https://www.dkrz.de/redmine/projects/yaxt, accessed 20 July 2018.

[20] W. Deconinck, P. Bauer, M. Diamantakis, M. Hamrud, C. Kühnlein, P. Maciel, G. Mengaldo, T. Quintino, B. Raoult, P. K. Smolarkiewicz, N. P. Wedi, Atlas : A library for numerical weather prediction and climate modelling, Comput. Phy. Comm. 220 (2017) 188 - 204. doi:https://doi.org/10.1016/j.cpc.2017.07.006.

URL http://www.sciencedirect.com/science/article/pii/ S0010465517302138

[21] XIOS wiki, http://forge.ipsl.jussieu.fr/ioserver, accessed 16 July 2018.

[22] S. V. Adams, O. Abramkina, Y. Meurdesoif, M. Rezny, Parallel IO in the LFRic infrastructure, in: S. Bassini, M. Danelutto, P. Dazzi, G. R. Joubert, F. Peters (Eds.), Parallel Computing is Everywhere, IOS Press, 2018, pp. 485-494. 
[23] CF Conventions and Metadata, http://cf conventions.org/, accessed 12 October 2018.

[24] UGRID Convention V1.0, http://ugrid-conventions.github.io/ugridconventions, accessed 16 July 2018.

[25] R. W. Ford, A. R. Porter, PSyclone: a domain-specific compiler for finiteelement/volume/difference earth-system modelsIn preparation.

[26] C. Bertolli, A. Betts, G. Mudalige, M. Giles, P. Kelly, Design and performance of the op2 library for unstructured mesh applications, in: M. e. a. Alexander (Ed.), Euro-Par 2011: Parallel Processing Workshops, Vol. 7155 of Lecture Notes in Computer Science, Springer Berlin Heidelberg, 2012, pp. 191-200.

[27] F. Rathgeber, G. R. Markall, L. Mitchell, N. Loriant, D. A. Ham, C. Bertolli, P. H. J. Kelly, Pyop2: A high-level framework for performance-portable simulations on unstructured meshes, in: Proceedings of the 2012 SC Companion: High Performance Computing, Networking Storage and Analysis, SCC '12, IEEE Computer Society, Washington, DC, USA, 2012, pp. 1116-1123. doi:10.1109/SC.Companion.2012.134.

URL http://dx.doi.org/10.1109/SC.Companion.2012.134

[28] T. Gysi, C. Osuna, O. Fuhrer, M. Bianco, T. Schulthess, in: Proceedings of the International Conference for High Performance Computing, Networking, Storage and Analysis, SC 15, ACM, 2015, pp. 1-41.

[29] F. Rathgeber, D. A. Ham, L. Mitchell, M. Lange, F. Luporini, A. T. McRae, G.T. Bercea, G. R. Markall, P. H. Kelly, Firedrake: automating the finite element method by composing abstractions, Submitted to ACM TOMSarXiv:1501.01809. URL http://arxiv.org/abs/1501.01809

[30] A. Logg, K.-A. Mardal, G. N. Wells, et al., Automated Solution of Differential Equations by the Finite Element Method, Springer, 2012. doi:10.1007/978-3642-23099-8. 
[31] Kokkos GitHub repository, https://github.com/kokkos/kokkos, accessed 30 July 2018.

[32] D. Medina, A. St-Cyr, T. Warburton, High-order finite-differences on multithreaded architectures using occa., in: R. Kirby, M. Berzins, J. Hesthaven (Eds.), Spectral and High Order Methods for Partial Differential Equations ICOSAHOM 2014., Vol. 106 of Lecture Notes in Computational Science and Engineering, Springer, 2015, pp. 365-373.

[33] V. Clement, S. Ferrachat, O. Fuhrer, X. Lapillonne, C. E. Osuna, R. Pincus, J. Rood, W. Sawyer, The claw dsl: Abstractions for performance portable weather and climate models, in: Proceedings of the Platform for Advanced Scientific Computing Conference, PASC '18, ACM, New York, NY, USA, 2018, pp. 2:12:10. doi:10.1145/3218176.3218226.

URL http://doi.acm.org/10.1145/3218176.3218226

[34] A. R. Porter, R. W. Ford, M. Ashworth, G. D. Riley, M. Modani, Towards compiler-agnostic performance in finite-difference codes, in: G. R. Joubert, H. Leather, M. Parsons, F. Peters, M. Sawyer (Eds.), Parallel Computing: On the Road to Exascale, Vol. 27, IOS Press, Amsterdam, New York, Tokyo, 2016, pp. 647-658.

[35] A. Porter, J. Appleyard, M. Ashworth, R. Ford, J. Holt, H. Liu, G. Riley, Portable multi- and many-core performance for finite difference codes; Application to the free-surface component of NEMO, Geosci. Model Dev. Discussions 2017 (2017) 1-27. doi:10.5194/gmd-2017-150.

URL https: //www.geosci-model-dev-discuss.net/gmd-2017-150/

[36] S. Börm, R. Hiptmair, Analysis of tensor product multigrid, Numer. Algorithms 26 (3) (2001) 219-234.

[37] S. Balay, W. D. Gropp, L. C. McInnes, B. F. Smith, Efficient management of parallelism in object oriented numerical software libraries, in: E. Arge, A. M. Bruaset, H. P. Langtangen (Eds.), Modern Software Tools in Scientific Computing, Birkhäuser Press, 1997, pp. 163-202. 
[38] S. Balay, S. Abhyankar, M. F. Adams, J. B. P. Brune, K. Buschelman, L. Dalcin, V. Eijkhout, W. D. Gropp, D. Kaushik, M. G. Knepley, D. A. May, L. C. McInnes, R. T. Mills, T. Munson, K. Rupp, P. Sanan, B. F. Smith, S. Zampini, H. Zhang, H. Zhang, PETSc Web page, http://www.mcs.anl.gov/petsc, accessed 25 July 2018.

URL http: //www.mcs.anl.gov/petsc

[39] M. Blatt, P. Bastian, The iterative solver template library, in: B. Kågström, E. Elmroth, J. Dongarra, J. Waśniewski (Eds.), Applied Parallel Computing. State of the Art in Scientific Computing, Springer, 2007, pp. 666-675. doi:10.1007/978-3-540-75755-9_82.

[40] B. Cockburn, J. Gopalakrishnan, A characterization of hybridized mixed methods for second order elliptic problems, SIAM Journal on Numerical Analysis 42 (1) (2004) 283-301.

[41] T. H. Gibson, L. Mitchell, D. A. Ham, C. J. Cotter, A domain-specific language for the hybridization and static condensation of finite element methods, CoRR abs/1802.00303. arXiv:1802.00303.

URL http: //arxiv.org/abs/1802.00303

[42] E. H. Müller, R. Scheichl, Massively parallel solvers for elliptic partial differential equations in numerical weather and climate prediction, Q. J. Roy. Meteorol. Soc. 140 (685) (2014) 2608-2624.

[43] A. Dedner, E. Müller, R. Scheichl, Efficient multigrid preconditioners for atmospheric flow simulations at high aspect ratio, Int. J. Numer. Meth. F. 80 (1) (2016) 76-102.

[44] L. Mitchell, E. H. Müller, High level implementation of geometric multigrid solvers for finite element problems: Applications in atmospheric modelling, J. Comput. Phys. 327 (2016) 1-18.

[45] P. A. Ullrich, T. Melvin, C. Jablonowski, A. Staniforth, A proposed baroclinic 
wave test case for deep- and shallow-atmosphere dynamical cores, Q. J. Roy. Meteorol. Soc. 140 (682) (2013) 1590-1602. doi:10.1002/qj.2241.

[46] O. Fuhrer, C. Osuna, X. Lapillonne, T. Gysi, B. Cumming, M. Bianco, A. Arteaga, T. Schulthess, Towards a performance portable, architecture agnostic implementation strategy for weather and climate models, Supercomp. Front. Innov. 1 (1). doi:10.14529/jsfi140103.

URL http://superfri.org/superfri/article/view/17

[47] ENIAC project website, https://www.pasc-ch.org/projects/20172020/eniac-enabling-the-icon-model-on-heterogeneousarchitectures/, accessed 30 July 2018.

[48] E3SM project website, https://climatemodeling.science.energy.gov/ projects/energy-exascale-earth-system-model, accessed 30 July 2018. 